\title{
How Chromosome Mis-Segregation Leads to Cancer: Lessons from BubR1 Mouse Models
}

\author{
Hyunsook Lee*
}

\begin{abstract}
Alteration in chromosome numbers and structures instigate and foster massive genetic instability. As Boveri has seen a hundred years ago (Boveri, 1914; 2008), aneuploidy is hallmark of many cancers. However, whether aneuploidy is the cause or the result of cancer is still at debate. The molecular mechanism behind aneuploidy includes the chromosome mis-segregation in mitosis by the compromise of spindle assembly checkpoint (SAC). SAC is an elaborate network of proteins, which monitor that all chromosomes are bipolarly attached with the spindles. Therefore, the weakening of the SAC is the major reason for chromosome number instability, while complete compromise of SAC results in detrimental death, exemplified in natural abortion in embryonic stage. Here, I will review on the recent progress on the understanding of chromosome missegregation and cancer, based on the comparison of different mouse models of BubR1, the core component of SAC.
\end{abstract}

\section{THE ROLE OF BUbR1 IN MITOSIS}

BubR1 is an orthologue of yeast Mad3 that binds and inhibits the multisubunit E3 ligase APC/C in SAC signaling (Chao et al., 2012; Taylor et al., 1998). As illustrated in Fig. 1, human BubR1 is $\sim 125 \mathrm{kDa}$ protein, composed of 1,050 amino acids. Yeast Mad3 and mammalian BubR1 share homology at the $\mathrm{N}$ terminus: TPR (tetratricopeptide repeat) domain is required for recruitment to kinetochore by binding to Blinkin/KNL1 (Kiyomitsu et al., 2007; Krenn et al., 2012). KNL1 is a core component of KMN network, essential for kinetochore-microtubule interaction for chromosome alignment (Takeuchi and Fukagawa, 2012; Varma and Salmon, 2012); GLEBS (Gle-2-binding sequence) motif is for Bub3 binding (Larsen et al., 2007); KEN boxes and $\mathrm{D}$-box found in mammalian BubR1 are the degrons required for binding to Cdc20 and APC/C (Burton and Solomon, 2007; LaraGonzalez et al., 2011; Tian et al., 2012).

Department of Biological Sciences and the Institute of Molecular Biology and Genetics, Seoul National University, Seoul 151-742, Korea

*Correspondence: HL212@snu.ac.kr

Received 22 August, 2014; accepted 25 August, 2014; published online 26 September, 2014

Keywords: aneuploidy, BubR1, BubR1 acetylation, cancer, chromosome mis-segregation, mouse
Unlike yeast Mad3, mammalian BubR1 has kinase domain similar to Bub1 at the C-terminus (Fig. 1). For a number of years, significant efforts were made to reveal the substrates of BubR1 kinase. An interesting idea was that CENP-E, the plus enddirected microtubule motor, binds to BubR1 and activates the kinase activity for BubR1 (Mao et al., 2003; 2005). However, numerous following works failed to confirm the kinase activity of BubR1. Recently, it was suggested that the C-terminal kinase domain of BubR1 is a pseudo-kinase domain, resulting from the amplification from Bub1, and thus BubR1 does not possess any kinase activity (Suijkerbuijk et al., 2012a).

Mitotic kinases such as CDK1, Plk1, and Aurora B phosphorylate BubR1 at distinct sites. The outcome of the phosphorylation at each site is not fully understood, but is expected to be crucial in mitotic checkpoint signaling. BubR1 has two distinct functions in mitosis: regulation of chromosome congression and SAC (Elowe et al., 2010). Phosphorylation of BubR1 at serines and threnines of T680, T620, D670, D676, T680 are involved in tension sensing, kinetochore-microtubule interaction, and mitotic exit (Elowe et al., 2007; 2010; Huang et al., 2008; Suijkerbuijk et al., 2012a). Comparing the structure of yeast Mad3 with mammalian BubR1, it appears that the spindle checkpoint regulation may be a more ancient function and the role in the stabilization of kinetochore-microtubule attachment has evolved in vertebrates (Fig. 1). Apparently, the KARD domain, constituted of many Plk1-mediated phosphorylation sites are required for binding to PP2A-B56 $\alpha$, counteracting the excessive Aurora B kinase activity for chromosome alignment in metaphase (Kruse et al., 2013a; Suijkerbuijk et al., 2012a).

\section{BubR1 IN SAC ACTIVATION, MAINTENANCE, AND SILENCING}

Treatment of microtubule poisons result in hyperphosphorylation of BubR1, indicating that phosphorylations at multiple sites are important in exhibiting mitotic functions of BubR1. In addition to phosphorylation, BubR1 is acetylated at lysine 250 in prometaphase (Choi et al., 2009). Acetylated BubR1 functions as an inhibitor of $\mathrm{APC} / \mathrm{C}$ and the deacetylated BubR1 becomes the substrate of APC/C. Polyubiquitination and subsequent degradation of deacetylated BubR1 are followed by cyclin B destruction, leading to anaphase onset and mitotic exit. When acetylationmimetic mutant of BubR1 is expressed, it blocks the anaphase onset. On the contrary, the expression of acetylation-deficient form allows shortening of mitotic timing with unequal chro- 

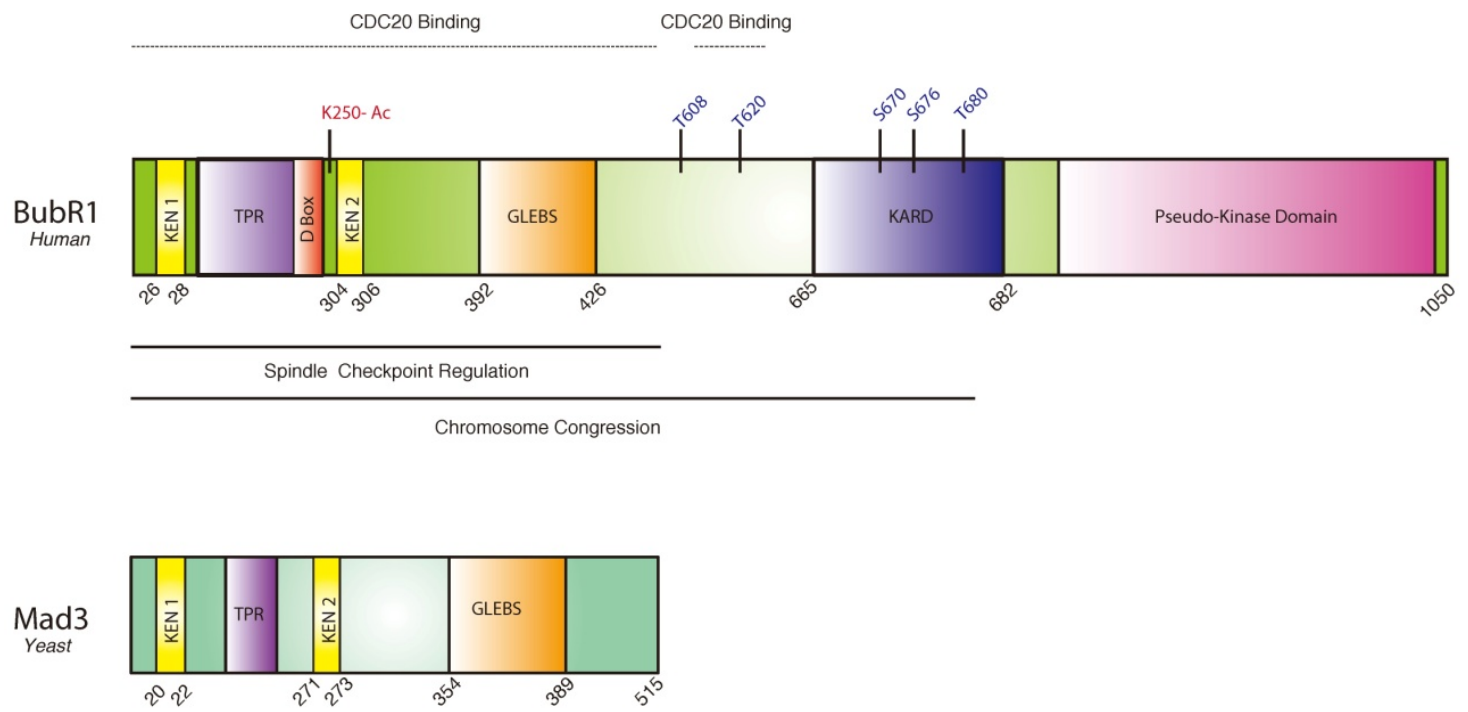

Fig. 1. Schematic illustration of human BubR1 and its ortholgue yeast Mad3. Functional domains, suggested functions, and reported phosphorylation and acetylation sites are marked.

mosome segregation. The result indicates that acetylation/deacetylation of BubR1 determines the mitotic timing and modulates APC/C activity. Thus, BubR1 acetylation is a crucial part of SAC signaling (Choi et al., 2009).

SAC activity can be divided into two phases: SAC signal generation and maintenance of SAC. First, Mad1 and Mad2 are recruited to unattached kinetochores. Then, Mad2 undergoes conformational change from open Mad2 (O-Mad2) to closed Mad2 (C-Mad2). C-Mad2 then binds to Cdc20, promoting the mitotic checkpoint complex (MCC) formation (Musacchio and Salmon, 2007), composed of Mad2, BubR1, Bub3, Cdc20. It is now known that Mad2 only loads Cdc20 to BubR1 and BubR1 is the true inhibitor for APC/C-Cdc20 (Han et al., 2013; Nilsson et al., 2008). MCC formation is thought to be the key to sustain SAC by titrating out Cdc20 from APC/C (Musacchio and Salmon, 2007; Sudakin et al., 2001), and also continuously ubiquitinating and degrading Cdc20 when SAC is on (Nilsson et al., 2008). Thus, MCC disassembly is crucial in checkpoint silencing.

Progress has been made recently on the understanding of how MCC disassembly is promoted, leading to checkpoint silencing. APC15, one of the components of APC/C mediates the autoubiquitination of cdc20, resulting in the degradation and disassembly of MCC (Foster and Morgan, 2012; Mansfeld et al., 2011; Uzunova et al., 2012).

Deacetylation of BubR1 (Choi et al., 2009) leads to ubiquitination by $\mathrm{APC} / \mathrm{C}$ and degradation of it, thus can also participate in the disassembly of MCC. Indeed, it was shown that cells isolated from the mouse heterozygous of acetylationdeficient allele $(K 243 R /+)$ failed to retain MCC upon nocodazole treatment, whereas the checkpoint signal generation, measured by Mad1/Mad2 localization at unattached kinetochores were intact. These results suggest that the early onset destruction of acetylation-deficient BubR1 may serve as the cue to disassembly of MCC (Park et al., 2013), and checkpoint silencing.

The role of $\mathrm{p} 31^{\text {comet }}$ has been identified in SAC silencing: it binds to C-Mad2-Cdc20 complex, thereby titrating out Mad2, promoting the disassembly of MCC (Miniowitz-Shemtov et al., 2012; Teichner et al., 2011; Westhorpe et al., 2011; Yun et al., 2007). However, an elegant biochemical study have shown that it is BubR1-Cdc20 complex that truly inhibits APC/C activity, and extracting Mad2 from the complex does not inhibit APC/C to a potent level, suggesting that the role of $\mathrm{p} 31^{\text {comet }}$ in SAC silencing may be secondary in SAC silencing (Han et al., 2013).

\section{FUNCTIONS OF BUbR1 IN CHROMOSOME-SPINDLE ATTACHMENT}

Loss of BubR1 results in the failure of the stable maintenance of chromosome-spindle attachment (Lampson and Kapoor, 2005). LosS of CENP-E, the plus end-directed motor protein that binds to BubR1 at kinetochore, leads to the increase of monotelic chromosomes near the pole (Kapoor et al., 2006). When compared to the loss of CENP-E, BubR1 loss leads to a more profound defects in chromosome congression, indicating a direct role of BubR1 in kinetochore-microtubule (KT-MT) interaction than CENP-E. Furthermore, impaired kinetochore-microtubule attachment is restored in BubR1-depleted cells when Aurora B activity is inhibited, suggesting that BubR1 antagonizes Aurora B's activity in KT-MT attachment (Lampson and Kapoor, 2005).

Erroneous microtubule attachment is corrected by the activity of Aurora B. Located at the inner kinetochore, Aurora B phosphorylates multiprotein complexes at the outer kinetochore, the KMN network (KNL1-Mis12-Ndc80) and Sca1-Dam complexes, destabilizing the kinetochore-microtubule attachments (Chan et al., 2012; Cheeseman et al., 2006; Welburn et al., 2010). Once amphitelic attachment is achieved, Aurora B activity must be counteracted to stabilize KT-MT attachment and chromosome alignment at the metaphase plate (congression). In brief, KT-MT attachment and SAC are well harmonized in mitosis by the activity of Aurora B: SAC is on when chromosomes are not attached to microtubules in a bipolar manner (Aurora $B$ active); and turns off when chromosomes-spindle attachment is satisfied (Aurora $B$ inactive). Therefore, phosphatases PP1 (Kim et al., 2010) and PP2A (Tanno et al., 2010) have been suggested to antagonize activity of Aurora $B$ to promote chromosome congression.

BubR1 recruits B56 $\alpha$ subunit of PP2A to the kinetochore, contributing to stable KT-MT attachment (Kruse et al., 2013b; Suijkerbuijk et al., 2012b). Notably, tension-sensitive pho- 
Table 1. Different BubR1 mouse models and their characteristics

\begin{tabular}{|c|c|c|c|}
\hline Strategy & Allele & Phenotype & Reference \\
\hline \multirow{3}{*}{$\begin{array}{l}\text { Targeted disruption of the BubR1 } \\
\text { locus between exon } 1 \text { and } 2 \\
\text { by gene-trapping method }\end{array}$} & BubR1 ${ }^{-1}$ & $\begin{array}{l}\text { Failed to survive beyond day } 8.5 \text { in utero as a result } \\
\text { of extensive apoptosis }\end{array}$ & \\
\hline & $B u b R 1^{+/}$ & $\begin{array}{l}\text {-Splenomegaly and abnormal megakaryopoiesis } \\
\text { coupled with decreased erythropoiesis } \\
\text {-When challenged with azoxymethane (AOM), } \\
\text { develop lung and intestinal adenocarcinomas }\end{array}$ & Wang et al., 2004 \\
\hline & $B u b R 1^{+/} ; A p c^{M i n /+}$ & $\begin{array}{l}\text { Develops ten-times more colonic tumor masses } \\
\text { than ApcMin/+ with higher grade polyps; yet } \\
\text { fewer polyps in small intestines. }\end{array}$ & Rao et al., 2005 \\
\hline $\begin{array}{l}\text { Hypomophic BubR1 expression: } \\
\text { The mutant allele was engi- } \\
\text { neered to generate abnormal } \\
\text { splicing. The resulting mutant } \\
\text { mRNA cannot produce BubR1 } \\
\text { protein. }\end{array}$ & $B u b 1 b^{H / H}$ & $\begin{array}{l}\text {-Aneuploidy } \\
\text {-Features of aging: short lifespan, cachectic dwarf- } \\
\text { ism, lordokyphosis, cataracts, loss of } \\
\text { subcutaneous fat and impaired wound healing } \\
\text {-Infertile } \\
\text {-No spontaneous tumorigenesis }\end{array}$ & Baker et al., 2004 \\
\hline $\begin{array}{l}\text { Homologous Recombination } \\
\text { mediated insertion of GTTA } \\
\text { squence in the murine BubR1 } \\
\text { gene at position } 2178\end{array}$ & $B u b R 1^{G T A /+}$ & $\begin{array}{l}\text {-Mimics nonsense mutation 2211insGTTA found in } \\
\text { MVA patients } \\
\text {-Mild aneuploidy } \\
\text {-Reduced life span and carry age-related pheno- } \\
\text { types: loss of skeletal muscle and fat } \\
\text {-Increased incidence of lung tumor upon DMBA } \\
\text { treatment }\end{array}$ & Wijshake et al., 2012 \\
\hline \multirow[t]{2}{*}{$\begin{array}{l}\text { Substitution of acetylation site } \\
\mathrm{K} 243 \text { to arginine in BubR1 } \\
\text { allele by homologous recom- } \\
\text { bination-mediated knock-in } \\
\text { method }\end{array}$} & BubR1 ${ }^{K 243 R / K 243 R}$ & $\begin{array}{l}\text { Embryonic lethal at E6.5 } \\
\text {-Heterozygous mutants exhibit spontaneous } \\
\text { tumorigenesis, one year after birth }\end{array}$ & \\
\hline & $\begin{array}{c}B u b R 1^{K 243 R /+} \\
(K 243 R /+)\end{array}$ & $\begin{array}{l}\text {-Tumor incidence }>40 \% \\
\text {-No developmental defect } \\
\text {-No aging phenotype } \\
\text {-Massive chromosome mis-segregation due to } \\
\text { impaired KT-MT attachment combined with } \\
\text { weakened SAC }\end{array}$ & Park et al., 2013 \\
\hline
\end{tabular}

sphorylations by Plk1 at the KARD domain (S670, S676, T680) are required for binding PP2A-B56 $\alpha$ to BubR1 at kinetochore (Fig. 1). PP2A activity at the kinetochore antagonizes the excessive activity of Aurora B in disassembly of microtubules at the kinetochore, thus stabilizing KT-MT attachments and achieving chromosome congression (Kruse et al., 2013b; Suijkerbuijk et al., 2012b; Xu et al., 2013). Collectively, BubR1 links KT-MT attachment to SAC signaling.

BubR1 acetylation at $\mathrm{K} 250$ is also required for recruiting PP2A-B56 $\alpha$ to the kinetochore. Interaction between BubR1 and PP2A-B56 $\alpha$ is acetylation-dependent, and cells isolated from the mice heterozygous for acetylation-deficient BubR1 (K243R/+) exhibit severe problems in chromosome congression (Park et al., 2013). How acetylation at K250 is coordinated with Plk1's phosphorylation at KARD domain is not known. Nevertheless, the result suggests an interesting hypothesis that there may be an acetylation-phosphorylation code in sensing KT-MT attachments to SAC signaling, and finally to SAC silencing.

\section{BUbR1 MUTATION IN HUMAN DISEASE}

Mosaic Variegated Aneuploidy (MVA) and/or Premature chromatid separation (PCS) are rare genetic disorders that display aneuploidy and premature sister chromatid separations (PMSC) in metaphase chromosome spreads. MVA patients are characterized by severe microcephaly, growth defects, mental retardation, and neoplasia (Jacquemont et al., 2002; Kawame et al., 1999; Matsuura et al., 2000).

Notably, BubR1 mutation is associated with MVA with cancer predisposition (de Voer et al., 2011; Suijkerbuijk et al., 2010). However, it is unclear whether specific mutations are linked with distinct phenotypes in MVA of heterologous symptoms. Taken together with the BubR1 mutations found in somatic cancers (Bolanos-Garcia and Blundell, 2011), it is noteworthy that dysfunctional BubR1 contributes to tumorigenesis.

\section{BUbR1 MOUSE MODELS IN CANCER PREDISPOSITION}

Despite the frequent aneuploidy in human cancers, mutations in SAC genes are rare. The reason for this may be that SAC is 
essential in cell proliferation, therefore is detrimental when deleted. Indeed, homozygous deletions of genes coding for Mad2 (Dobles et al., 2000), Bub1 (Jeganathan et al., 2007), BubR1 (Wang et al., 2004) in mice exhibit early embryonic lethality, indicating that severe mitotic failure are indeed detrimental.

Mice knockout of BubR1 allele are lethal in early embryonic stage; heterozygous mice are viable and display megakaryopoiesis with aneuploidy but not spontaneous cancers (Wang et al., 2004). Paradoxically, gradual reduction of BubR1 level in mice of hypomorphic BubR1 allele exhibit senescence and premature aging but not spontaneous cancer (Baker et al., 2004). Apparently, both haploinsufficient (Wang et al., 2004) and hypomorphic BubR1 allele display aneuploidy, indicating that aneuploidy alone does not impose on tumorigenesis. Notably, mice heterozygous of CENPE-null allele (Weaver et al., 2007) imply that aneuploidy can be oncogenic to a certain degree, but is tumor suppressive when combined with more genetic insults. Indeed, aneuploidy in mammalian cells induce proliferation defects and metabolic abnormalities, suggesting that the burden of having to produce too much nucleic acids and proteins are toxic to cellular fitness (Williams et al., 2008), consistent with the phenotypes observed from Down's syndrome (Table 1 ).

However, when BubR1 haploinsufficient mice are crossed to $A P C^{\mathrm{Min} /+}$ mice, tumorigenesis in the large intestine increases to ten-fold, with the decrease of the polyps in the small intestines (Rao et al., 2005), suggesting that there is more than aneuploidy in BubR1 mutation, compared to CENP-E haploinsufficiency. It is worth mentioning that the depletion of BubR1 has far more profound effects in chromosome congression, compared to CENPE depletion, as mentioned above (Lampson and Kapoor, 2005).

Reduction of BubR1 protein level predisposes to early onset aging in mice (Baker et al., 2004), which is also observed in the monoallelic insertional mutation of BubR1 at nucleotide 2118 position (Wijshake et al., 2012). This allale (BubR1 $1^{G T A /+}$ ) was created after the genetic mutation in one of the MVA patients. However, the mutation lies after the KARD domain (Fig. 1) and affects the C-terminus pseudokinase domain, which might not have crucial functions in SAC. Nevertheless, BubR $1^{\mathrm{H} / H}$ and $B u b R 1^{G T T A+}$ mice links BubR1 mutation with aging-related MVA phenotype, albeit the absence of tumor formation (Summarized in Table 1).

Notably, cancer predisposition is observed in mice heterozygous of acetylation-deficient BubR1 allele (K243R/+): K243R/+ mice are succumbed to high incidence of spontaneous tumorigenesis, most abundantly lymphoma (Park et al., 2013). In fact, this is the first mouse model to show that a single mutation in SAC components can lead to high-incidence spontaneous tumorigenesis.

It is important to speculate the difference of $K 243 R /+$ mice from those of other BubR1 mouse models in cancer predisposition. Critical difference may rely on the fact that acetylation of K250 (K243 in mice) has dual roles: one in stable chromosomespindle attachment and the other in the stable maintenance of MCC until checkpoint is satisfied. Homozygous mutations are detrimental that it exhibits embryonic lethality at embryonic day 6 , even before the homozygous deletion of BubR1 allele (Table 1).

In K243R/+ heterozygous cells, the wild-type and mutant protein co-exist in 1:1 ratio; K243R protein localizes to the kinetochore as much as the wild-type BubR1 does, inhibiting the recruitment of PP2A-B56 $\alpha$ to kinetochores, thus $K 243 R$ allele is a dominant negative allele. In comparison, BubR1 in BubR1 ${ }^{+/}$cells localized more to the kinetochores with the decrease of the cytosolic portion, therefore the defect was not as severe as $K 243 R /+$, explaining the absence of spontaneous tumorigenesis in $B u b R 1^{+/}$mice. It is worth mentioning that the acetylation of
BubR1 at K250 requires BRCA2, hence BubR1 acetylation is a tumor suppressive mechanism (Choi et al., 2012). K243R/+ mice represent the BRCA2-deficiency in mitotic failure and tumorigeneis.

\section{CONCLUSION}

What makes $K 243 R /+$ mice tumor-prone and others don't? It is conceivable to think that the massive chromosome missegregation resulting from the combined effects of failure in KTMT attachment and weakened SAC instigated genetic instability, thus cancer, in K243R/+ mice. As observed from BubR1 haploinsufficiency, cells try to cope with the reduced level of BubR1 in mitosis. However, the aging phenotypes in hypomorphic BubR1 mouse and the BubR1 $1^{G T A+}$ mice suggest that reduced level of BubR1 may have effects in interphase, leading to senescence and aging.

Recently, it was shown that BubR1 acetylation status modulates the outgrowth of dendrite in neuronal cells (Watanabe et al., 2014) and also the lifespan (North et al., 2014), indicating that BubR1 has many more functions than unerstood. Nevertheless, lessons from $K 243 R /+$ mice indicate that BubR1 mutations linked with its critical functions in mitosis can predispose to tumorigenesis. It will be important to assign different human genetic mutations with different functions of BubR1.

\section{ACKNOWLEDGMENTS}

I thank I. Park for help with preparing the manuscript. I am indebted to all members of Lee lab for their support. This work was supported by the Korean NRF (2013 R1A2A1A02009184 \& 2012M3A9A8052645) and by the Cancer Control program from the Korean Ministry of Health and Welfare (1220210). I apologize that numerous excellent works could not be cited due to space limitation.

\section{REFERENCES}

Baker, D.J., Jeganathan, K.B., Cameron, J.D., Thompson, M. Juneja, S., Kopecka, A., Kumar, R., Jenkins, R.B., de Groen, P.C., Roche, P., et al. (2004). BubR1 insufficiency causes early onset of aging-associated phenotypes and infertility in mice. Nat. Genet. 36, 744-749.

Bolanos-Garcia, V.M., and Blundell, T.L. (2011). BUB1 and BUBR1: multifaceted kinases of the cell cycle. Trends Biochem. Sci. 36, $141-150$

Boveri, T. (1914). Zur Frage der Entstehung maligner Tumorer (The origin of malignant tumors). (Jena: Gustav Fischer).

Boveri, T. (2008). Concerning the origin of malignant tumours by Theodor Boveri. Translated and annotated by Henry Harris. J. Cell Sci. 121 (Suppl 1), 1-84.

Burton, J.L., and Solomon, M.J. (2007). Mad3p, a pseudosubstrate inhibitor of APCCdc20 in the spindle assembly checkpoint. Genes Dev. 21, 655-667.

Chan, Y.W., Jeyaprakash, A.A., Nigg, E.A., and Santamaria, A. (2012). Aurora B controls kinetochore-microtubule attachments by inhibiting Ska complex-KMN network interaction. J. Cell Biol. 196, 563-571.

Chao, W.C., Kulkarni, K., Zhang, Z., Kong, E.H., and Barford, D. (2012). Structure of the mitotic checkpoint complex. Nature 484, 208-213.

Cheeseman, I.M., Chappie, J.S., Wilson-Kubalek, E.M., and Desai, A (2006). The conserved KMN network constitutes the core microtubule-binding site of the kinetochore. Cell 127, 983-997.

Choi, E., Choe, H., Min, J., Choi, J.Y., Kim, J., and Lee, H. (2009). BubR1 acetylation at prometaphase is required for modulating APC/C activity and timing of mitosis. EMBO J. 28, 2077-2089.

Choi, E., Park, P.G., Lee, H.O., Lee, Y.K., Kang, G.H., Lee, J.W., Han W., Lee, H.C., Noh, D.Y., Lekomtsev, S., et al. (2012). BRCA2 fine-tunes the spindle assembly checkpoint through reinforcement of BubR1 acetylation. Dev. Cell 22, 295-308. 
de Voer, R.M., Hoogerbrugge, N., and Kuiper, R.P. (2011). Spindleassembly checkpoint and gastrointestinal cancer. N Engl. J. Med. 364, 1279-1280.

Dobles, M., Liberal, V., Scott, M.L., Benezra, R., and Sorger, P.K. (2000). Chromosome missegregation and apoptosis in mice lacking the mitotic checkpoint protein Mad2. Cell 101, 635-645.

Elowe, S., Hummer, S., Uldschmid, A., Li, X., and Nigg, E.A. (2007). Tension-sensitive Plk1 phosphorylation on BubR1 regulates the stability of kinetochore microtubule interactions. Genes Dev. 21, 2205-2219.

Elowe, S., Dulla, K., Uldschmid, A., Li, X., Dou, Z., and Nigg, E.A (2010). Uncoupling of the spindle-checkpoint and chromosomecongression functions of BubR1. J. Cell Sci. 123, 84-94.

Foster, S.A., and Morgan, D.O. (2012). The APC/C subunit Mnd2/Apc15 promotes Cdc20 autoubiquitination and spindle assembly checkpoint inactivation. Mol. Cell 47, 921-932.

Han, J.S., Holland, A.J., Fachinetti, D., Kulukian, A., Cetin, B., and Cleveland, D.W. (2013). Catalytic assembly of the mitotic checkpoint inhibitor BubR1-Cdc20 by a Mad2-induced functional switch in Cdc20. Mol. Cell 51, 92-104.

Huang, H., Hittle, J., Zappacosta, F., Annan, R.S., Hershko, A., and Yen, T.J. (2008). Phosphorylation sites in BubR1 that regulate kinetochore attachment, tension, and mitotic exit. J. Cell Biol. 183, 667-680.

Jacquemont, S., Boceno, M., Rival, J.M., Mechinaud, F., and David, A. (2002). High risk of malignancy in mosaic variegated aneuploidy syndrome. Am. J. Med. Genet. 109, 17-21; discussion 16.

Jeganathan, K., Malureanu, L., Baker, D.J., Abraham, S.C., and van Deursen, J.M. (2007). Bub1 mediates cell death in response to chromosome missegregation and acts to suppress spontaneous tumorigenesis. J. Cell Biol. 179, 255-267.

Kapoor, T.M., Lampson, M.A., Hergert, P., Cameron, L., Cimini, D. Salmon, E.D., McEwen, B.F., and Khodjakov, A. (2006). Chromosomes can congress to the metaphase plate before biorientation. Science 311, 388-391.

Kawame, H., Sugio, Y., Fuyama, Y., Hayashi, Y., Suzuki, H., Kurosawa, K., and Maekawa, K. (1999). Syndrome of microcephaly, Dandy-Walker malformation, and Wilms tumor caused by mosaic variegated aneuploidy with premature centromere division (PCD) report of a new case and review of the literature. J. Hum. Genet 44, 219-224.

Kim, Y., Holland, A.J., Lan, W., and Cleveland, D.W. (2010). Aurora kinases and protein phosphatase 1 mediate chromosome congression through regulation of CENP-E. Cell 142, 444-455.

Kiyomitsu, T., Obuse, C., and Yanagida, M. (2007). Human Blinkin/AF15q14 is required for chromosome alignment and the mitotic checkpoint through direct interaction with Bub1 and BubR1. Dev. Cell 13, 663-676.

Krenn, V., Wehenkel, A., Li, X., Santaguida, S., and Musacchio, A (2012). Structural analysis reveals features of the spindle checkpoint kinase Bub1-kinetochore subunit Knl1 interaction. J. Cell Biol. 196, 451-467.

Kruse, T., Zhang, G., Larsen, M.S., Lischetti, T., Streicher, W., Kragh Nielsen, T., Bjorn, S.P., and Nilsson, J. (2013a). Direct binding between BubR1 and B56-PP2A phosphatase complexes regulate mitotic progression. J. Cell Sci. 126, 1086-1092.

Kruse, T., Zhang, G., Larsen, M.S., Lischetti, T., Streicher, W., Nielsen, T.K., Bjorn, S.P., and Nilsson, J. (2013b). Direct binding between BubR1 and B56-PP2A phosphatase complexes regulate mitotic progression. J. Cell Sci.126, 1086-1092.

Lampson, M.A., and Kapoor, T.M. (2005). The human mitotic checkpoint protein BubR1 regulates chromosome-spindle attachments. Nat. Cell Biol. 7, 93-98

Lara-Gonzalez, P., Scott, M.I., Diez, M., Sen, O., and Taylor, S.S. (2011). BubR1 blocks substrate recruitment to the APC/C in a KEN-box-dependent manner. J. Cell Sci. 124, 4332-4345.

Larsen, N.A., Al-Bassam, J., Wei, R.R., and Harrison, S.C. (2007). Structural analysis of Bub3 interactions in the mitotic spindle checkpoint. Proc. Natl. Acad. Sci. USA 104, 1201-1206.

Mansfeld, J., Collin, P., Collins, M.O., Choudhary, J.S., and Pines, J. (2011). APC15 drives the turnover of MCC-CDC20 to make the spindle assembly checkpoint responsive to kinetochore attachment. Nat. Cell Biol. 13, 1234-1243.

Mao, Y., Abrieu, A., and Cleveland, D.W. (2003). Activating and silencing the mitotic checkpoint through CENP-E-dependent activation/inactivation of BubR1. Cell 114, 87-98.
Mao, Y., Desai, A., and Cleveland, D.W. (2005). Microtubule capture by CENP-E silences BubR1-dependent mitotic checkpoint signaling. J. Cell Biol. 170, 873-880.

Matsuura, S., Ito, E., Tauchi, H., Komatsu, K., Ikeuchi, T., and Kajii, T. (2000). Chromosomal instability syndrome of total premature chromatid separation with mosaic variegated aneuploidy is defective in mitotic-spindle checkpoint. Am. J. Hum. Genet. 67, 483-486.

Miniowitz-Shemtov, S., Eytan, E., Ganoth, D., Sitry-Shevah, D., Dumin, E., and Hershko, A. (2012). Role of phosphorylation of Cdc20 in p31(comet)-stimulated disassembly of the mitotic checkpoint complex. Proc. Natl. Acad. Sci. USA 109, 8056-8060.

Musacchio, A., and Salmon, E.D. (2007). The spindle-assembly checkpoint in space and time. Nat. Rev. Mol. Cell Biol. 8, 379-393

Nilsson, J., Yekezare, M., Minshull, J., and Pines, J. (2008). The APC/C maintains the spindle assembly checkpoint by targeting Cdc20 for destruction. Nat. Cell Biol. 10, 1411-1420.

North, B.J., Rosenberg, M.A., Jeganathan, K.B., Hafner, A.V. Michan, S., Dai, J., Baker, D.J., Cen, Y., Wu, L.E., Sauve, A.A., et al. (2014). SIRT2 induces the checkpoint kinase BubR1 to increase lifespan. EMBO J. 33, 1438-1453.

Park, I., Lee, H.O., Choi, E., Lee, Y.K., Kwon, M.S., Min, J., Park, P.G., Lee, S., Kong, Y.Y., Gong, G., et al. (2013). Loss of BubR1 acetylation causes defects in spindle assembly checkpoint signaling and promotes tumor formation. J. Cell Biol. 202, 295-309.

Rao, C.V., Yang, Y.M., Swamy, M.V., Liu, T., Fang, Y., Mahmood, R., Jhanwar-Uniyal, M., and Dai, W. (2005). Colonic tumorigenesis in BubR1+/-ApcMin/+ compound mutant mice is linked to premature separation of sister chromatids and enhanced genomic instability. Proc. Natl. Acad. Sci. USA 102, 4365-4370.

Sudakin, V., Chan, G.K., and Yen, T.J. (2001). Checkpoint inhibition of the APC/C in HeLa cells is mediated by a complex of BUBR1, BUB3, CDC20, and MAD2. J. Cell Biol. 154, 925-936.

Suijkerbuijk, S.J., van Osch, M.H., Bos, F.L., Hanks, S., Rahman, N., and Kops, G.J. (2010). Molecular causes for BUBR1 dysfunction in the human cancer predisposition syndrome mosaic variegated aneuploidy. Cancer Res. 70, 4891-4900.

Suijkerbuijk, S.J., van Dam, T.J., Karagoz, G.E., von Castelmur, E., Hubner, N.C., Duarte, A.M., Vleugel, M., Perrakis, A., Rudiger, S.G., Snel, B., et al. (2012a). The vertebrate mitotic checkpoint protein BUBR1 is an unusual pseudokinase. Dev. Cell 22, 13211329.

Suijkerbuijk, S.J., Vleugel, M., Teixeira, A., and Kops, G.J. (2012b). Integration of kinase and phosphatase activities by BUBR1 ensures formation of stable kinetochore-microtubule attachments. Dev. Cell 23, 745-755.

Takeuchi, K., and Fukagawa, T. (2012). Molecular architecture of vertebrate kinetochores. Exp. Cell Res. 318, 1367-1374.

Tanno, Y., Kitajima, T.S., Honda, T., Ando, Y., Ishiguro, K., and Watanabe, Y. (2010). Phosphorylation of mammalian Sgo2 by Aurora B recruits PP2A and MCAK to centromeres. Genes Dev. 24, 2169-2179.

Taylor, S.S., Ha, E. and McKeon, F. (1998). The human homologue of Bub3 is required for kinetochore localization of Bub1 and a Mad3/Bub1-related protein kinase. J. Cell Biol 142, 1-11.

Teichner, A., Eytan, E., Sitry-Shevah, D., Miniowitz-Shemtov, S., Dumin, E., Gromis, J., and Hershko, A. (2011). p31comet Promotes disassembly of the mitotic checkpoint complex in an ATPdependent process. Proc. Natl. Acad. Sci. USA 108, 3187-3192.

Tian, W., Li, B., Warrington, R., Tomchick, D.R., Yu, H., and Luo, X. (2012). Structural analysis of human Cdc20 supports multisite degron recognition by APC/C. Proc. Natl. Acad. Sci. USA 109, 18419-18424.

Uzunova, K., Dye, B.T., Schutz, H., Ladurner, R., Petzold, G., Toyoda Y., Jarvis, M.A., Brown, N.G., Poser, I., Novatchkova, M., et al. (2012). APC15 mediates CDC20 autoubiquitylation by APC/C(MCC) and disassembly of the mitotic checkpoint complex. Nat. Struct. Mol. Biol. 19, 1116-1123.

Varma, D., and Salmon, E.D. (2012). The KMN protein networkchief conductors of the kinetochore orchestra. J. Cell Sci. 125, 5927-5936.

Watanabe, Y., Khodosevich, K., and Monyer, H. (2014). Dendrite development regulated by the schizophrenia-associated gene FEZ1 involves the ubiquitin proteasome system. Cell Rep. 7, 552-564.

Weaver, B.A., Silk, A.D., Montagna, C., Verdier-Pinard, P., and Cleveland, D.W. (2007). Aneuploidy acts both oncogenically and 
as a tumor suppressor. Cancer Cell 11, 25-36.

Welburn, J.P., Vleugel, M., Liu, D., Yates, J.R., 3rd, Lampson, M.A. Fukagawa, T., and Cheeseman, I.M. (2010). Aurora B phosphorylates spatially distinct targets to differentially regulate the kinetochore-microtubule interface. Mol. Cell 38, 383-392.

Westhorpe, F.G., Tighe, A., Lara-Gonzalez, P., and Taylor, S.S (2011). p31comet-mediated extraction of Mad2 from the MCC promotes efficient mitotic exit. J. Cell Sci. 124, 3905-3916.

Wijshake, T., Malureanu, L.A., Baker, D.J., Jeganathan, K.B., van de Sluis, B., and van Deursen, J.M. (2012). Reduced life- and healthspan in mice carrying a mono-allelic BubR1 MVA mutation. PLoS Genet. 8, e1003138.
Williams, B.R., Prabhu, V.R., Hunter, K.E., Glazier, C.M., Whittaker, C.A., Housman, D.E., and Amon, A. (2008). Aneuploidy affects proliferation and spontaneous immortalization in mammalian cells. Science 322, 703-709.

Xu, P., Raetz, E.A., Kitagawa, M., Virshup, D.M., and Lee, S.H. (2013). BUBR1 recruits PP2A via the B56 family of targeting subunits to promote chromosome congression. Biol. Open 2, 479486.

Yun, M.Y., Kim, S.B., Park, S., Han, C.J., Han, Y.H., Yoon, S.H., Kim S.H., Kim, C.M., Choi, D.W., Cho, M.H., et al. (2007). Mutation analysis of p31comet gene, a negative regulator of Mad2, in human hepatocellular carcinoma. Exp. Mol. Med. 39, 508-513. 